\title{
Modelo de Calidad de E-learning para Instituciones de Educación Superior en Colombia
}

\author{
José F. Mejía ${ }^{(1)}$ y Diego López ${ }^{(2,3)}$ \\ (1) Facultad de Ciencias e Ingeniería, Universidad de Manizales, carrera 9 \# 19-03, Manizales-Colombia. \\ (e-mail: jfmejia@umanizales.edu.co) \\ (2) Facultad de Ciencias e Ingeniería, Universidad de Manizales, carrera 9 \# 19-03, Manizales-Colombia. \\ (e-mail: dlopez@umanizales.edu.co) \\ (3) Facultad de Administración, Departamento de Informática y computación, Universidad Nacional de \\ Colombia sede Manizales. (e-mail: dilopezca@unal.edu.co)
}

Recibido Sep. 1, 2015; Aceptado Oct. 29, 2015; Versión final Dic. 17, 2015, Publicado Abr. 2016

\begin{abstract}
Resumen
El objetivo del trabajo es presentar un modelo para determinar la calidad de procesos de e-learning para Instituciones de Educación Superior en Colombia. El modelo posee una estructura de interoperabilidad que permite la reutilización de materiales y metodologías, brindando medios tecnológicos para el desarrollo de ambientes adecuados de aprendizaje. Se considera también en la propuesta la adopción y adaptación de modelos de referencia, procesos de autoevaluación y regulación que integra estándares internacionales de calidad, con la política y legislación Colombiana. Finalmente, se incluye una marca de calidad que certifica procesos y productos, teniendo en cuenta características institucionales y contextuales. El modelo se validó en veintidós universidades colombianas, para las que se estableció un diagnóstico a través de encuestas, entrevistas y observación de campo. El estudio muestra la ausencia de criterios unificados o modelos para abordar procesos educativos virtuales.
\end{abstract}

Palabras clave: e-learning, estándares de calidad, autoevaluación, educación superior, aprendizaje virtual

\section{E-learning Quality Model for Higher Education Institutions in Colombia}

\begin{abstract}
The aim of the paper is to present the model to determine the quality of e-learning processes in higher education institutions in Colombia. The model has a structure of interoperability that allows the reuse of materials, methods and learning environments, providing technology for the development of appropriate learning environments. The proposal also considers the adoption and adaptation of reference models, processes of self-evaluation and regulations that meet international quality standards and Colombian law. Finally, a quality seal that certifies processes and products, taking into account institutional and contextual features. The model was validated in twenty two Colombian universities, for which a diagnosis through surveys, interviews and field data. The study shows the absence of unified criteria or models to address educational virtual processes.
\end{abstract}

Keywords: e-learning, quality standards, self-rating, higher education, virtual learning 


\section{INTRODUCCIÓN}

En los últimos 15 años los sistemas educativos universitarios han propiciado una serie de alternativas y propuestas para la educación utilizando el Internet como medio de comunicación y tratando de desplazar en un alto porcentaje la presencialidad del profesor en el aula de clase. Diferentes metodologías mediadas por las TIC han propiciado la aparición de un buen número de apuestas pedagógicas y didácticas alrededor de lo que hoy se llama el e-learning.

Hacia el año 2000 el auge de las llamadas empresas ".com" creó una burbuja que avizoró un cambio importante en el que las Universidades para la segunda década del siglo XXI tendrían implantados sus programas de formación en Internet y la educación presencial entraría en un segundo plano. Un ejemplo de esto lo constituyó la "Estrategia de Lisboa" (Sesión Especial del Consejo Europeo de Lisboa 23 y 24 de Marzo de 2000), con la que se planeó hacer de la economía europea, la más competitiva e inclusiva del mundo (Dondi, 2008), por ello la educación y en particular el e-learning, fueron parte de las prioridades de las agendas políticas de los tres primeros años del presente siglo. Con estos antecedentes la gran mayoría de esfuerzos de las IES (Instituciones de Educación Superior) se centraron en el desarrollo de un núcleo de producción de material educativo apoyado en las tecnologías emergentes y difundidas gracias a la Internet (Martínez et ál, 2015), pero como lo cuestiona Dondi (2008) ¿bajo qué criterios en cuanto a calidad y armonización ocurrían estos procesos?

Estos aspectos inicialmente fueron regulados por cada Institución de acuerdo con sus políticas académicas y administrativas, sin un juicio indicador o comparación unificada (Bates, 2007), situación que se presentó en las universidades que tradicionalmente ejercían liderazgo en una región o país, las demás iniciaron sus procesos años después. Puede decirse entonces que existen dos tendencias básicas en cuanto a la relación entre la determinación de la calidad del e-learning (Pons et ál, 2015; Sangrá, 2001): quienes la consideran un apéndice de la presencialidad y quienes la conciben como una actividad formativa. Otros autores coinciden también en que el e-learning debe ser tratado como un sistema de educación totalmente diferente a otras modalidades y por tanto su sistema de calidad debe ser también visto de igual forma (Barberá E., 2008; Duart y Lupiáñez, 2005).

Ante estas situaciones algunos grupos de interés empezaron a trabajar desde el 2003 en la estandarización y la armonización, primero de especificaciones y luego de estándares al respecto. Se destacaron por ejemplo iniciativas como SCORM (Sharable Content Object Reference Model), AICC (Aviation Industry Computed Based Training Comitee) e IMS (IMS Global Learning Consortium). La más popular y difundida fue SCORM, que se limitó sólo a un estándar para reutilizar los contenidos, lo que más adelante consolidó los "bancos de objetos de aprendizaje".

A mediados de la segunda década del siglo XXI, la realidad del e-learning es diferente a la de hace 10 años, las expectativas de crecimiento del 100\% pasaron a ser del 35\% (Fundación Europea para la Calidad del elearning, EFQUEL, 2008) y la visión del uso de las TIC para la formación en los diferentes sectores es distinta, tal vez podría decirse que han aprendido a integrar ambas formas y que los rumbos de los desarrollos en la Web han cambiado en los últimos cinco años; pero de todas formas el problema de la calidad subyace y es elemento decisorio a la hora de elegir un camino de formación.

Para las organizaciones que están trabajando la calidad, la idea siempre ha sido crear un lenguaje único y universal, los llamados grupos de interés quieren apuntar a los mismos objetivos en cuanto a calidad del aprendizaje, pero al final cada uno quiere de alguna manera hacer prevalecer los propios para la parte del mercado que manejan (Cardona y Sánchez, 2010; Dondi, 2008). En el caso de Colombia la situación de la calidad del e-learning no es distinta a muchos países latinoamericanos. En una investigación sobre calidad del e-learning realizada por el Ministerio de Educación Nacional (2007), se pudo concluir para la oferta de programas en modalidad e-learning, que: i) El énfasis está en las plataformas tecnológicas y en la generación de recursos únicamente; ii) La reflexión es escasa sobre los aspectos pedagógicos y comunicativos; iii) Hay limitaciones en la formación de docentes para esta modalidad; y iv) Ausencia de referentes de calidad.

Frente a estas situaciones de carácter global y nacional se hace indispensable generar un modelo o un marco de referencia que pueda servir como soporte para la oferta actual y futura de programas en modalidad e-learning, cubriendo las falencias en cuanto a los aspectos pedagógicos, comunicacionales y administrativos, en las Instituciones de Educación Superior de Colombia. 


\title{
ELEMENTOS PARA LA CONSTRUCCIÓN DE UN MODELO DE CALIDAD DE E-LEARNING
}

\author{
Interoperabilidad y Calidad \\ El sistema de calidad del proyecto se observar desde tres dimensiones fundamentales (Donabedian,1980):
}

Productos: que se entienden como los programas académicos y los servicios asociados a ellos, en las tres funciones sustantivas (docencia, investigación y proyección social).

Procesos: comprenden todos los procesos académicos y administrativos requeridos para el establecimiento de un programa académico o sus servicios asociados.

Potenciales: abarcan las posibles líneas futuras de desarrollo para nuevos programas académicos y servicios asociados

Un e-learning exitoso depende de la interoperabilidad para el desarrollo de la calidad a través de estándares internacionales. Se reconoce como la falencia en el e-learning en Colombia la falta de interoperabilidad, la ausencia del desarrollo y la adaptación o adopción de estándares de calidad; es por esto, que el trabajo sugiere que los centros de educación superior en Colombia deben partir de tres tipos de estándares: estándares técnicos, estándares de tecnología para el aprendizaje y estándares de calidad (De la Vega, 2015; Stracke, 2007)

\section{Aplicación de un modelo genérico de estándares}

Un elemento importante en la construcción de un modelo de calidad para el e-learning lo constituye la visión que las IES tenga sobre la manera en que puede aplicar, además de la normatividad vigente, los estándares de calidad a su proceso de e-learning (Sandoval, 2013; Pawlowski, 2008). El modelo genérico le permitirá a las IES adoptar y adaptar estándares discutidos internacionalmente a sus procesos de calidad y en particular a los de e-learning con base en tres dimensiones estructurales (Stracke, 2006; Pawlowski, 2007): una orientada a asegurar la interoperabilidad y el desarrollo de la calidad (tipo); otra enfocada al tipo de elearning y al sujeto del proceso (dominio); y una última dirigida a los medios utilizados para el proceso de enseñanza a aprendizaje (entidades).

La figura 1 muestra la manera en que pueden relacionarse estas tres dimensiones. Como si se tratara de un punto en el espacio del plano cartesiano, cada elemento de una dimensión puede asociarse con otro y formar una combinación que determina características importantes para el desarrollo de la calidad del proceso de formación. Por ejemplo, si se toma el dominio "didáctica" y se relaciona con la entidad "ambientes de aprendizaje" y a su vez se asocian con los estándares de nivel, podría formarse la relación didáctica-ambientes de aprendizaje-nivel de calidad. Esto equivale a relacionar en este caso los sistemas y métodos de enseñanza con la organización del espacio, disposición y distribución de los recursos, el manejo del tiempo y las interacciones que se dan entre los participante; y a su vez, con el nivel de calidad deseado.

\section{Adaptación y adopción de un modelo genérico de estándares}

Como se discute en el desarrollo del presente trabajo y de acuerdo con varios autores (Ehlers y Pawlowski, 2006; Hilera G. y Hoya M., 2010; Pawlolwski, 2008; Stracke, 2006; Bates, 2007) entre otros, además de la norma ISO/IEC 19796, los estándares y la regulación contienen elementos primordiales para el desarrollo de un e-learning de calidad, pero estos deben ser armonizados y normalizados si quieren usarse como base de un sistema de calidad. Se debe considerar además, que los estándares nacionales, los internacionales, la normatividad y los propios, alcanzan a ser un modelo de referencia que puede ser implementado en diferentes escenarios, pero deben ser adaptados en las IES antes de considerarse adoptados (Stracke, 2010; Cardona y Sánchez, 2010). Esto debe conducir a un modelo organizacional específico que contiene el proceso de adaptación, pero también parámetros para establecer una cultura de calidad en la Institución.

El sistema de aseguramiento de la calidad tiene un flujo de operación que posee la ventaja de permitir juicios de valor sobre programas o instituciones lo más objetivamente posible y dar las recomendaciones del caso para la retroalimentación y acompañamiento por parte del MEN (Ministerio de Educación Nacional) en todos los procesos académicos y administrativos del programa o la Institución.

\section{Sistema de Aseguramiento de la Calidad en Colombia}

El sistema está integrado por tres subsistemas: Subsistema de evaluación, Subsistema de información y Subsistema de fomento

Las instituciones son evaluadas en dos momentos principales, uno de carácter obligatorio, el de su creación (condiciones mínimas de calidad), y el otro voluntario, con la acreditación (condiciones de alta calidad). Para su creación deben demostrar el cumplimiento de los requisitos estipulados en el Decreto 1478 de 1994, para las instituciones privadas, y en la Ley 30 de 1992, artículos 58 a 60, para las públicas. 


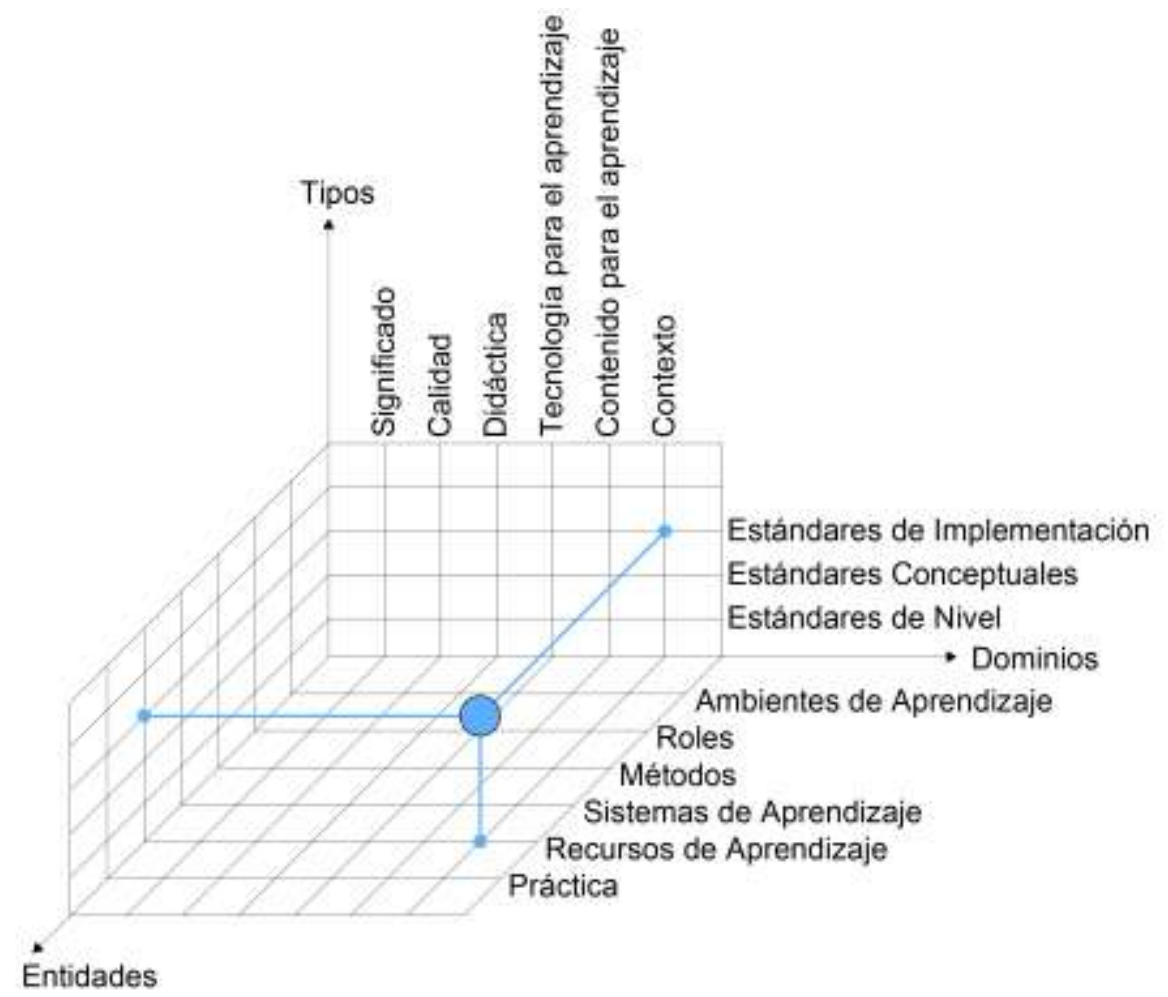

Fig. 1: Modelo genérico de estándares para el e-learning. Fuente: (Stracke, 2006)

\section{CONSTRUCCIÓN DE UN MODELO DE CALIDAD DE E-LEARNING}

De acuerdo a lo presentado en la revisión teórica del trabajo, el campo de estándares del e-learning, que deben soportar todo el proceso del ciclo de vida de un proyecto académico de educación superior se debe agrupar en cuatro áreas: análisis y diseño, concepción y desarrollo, proceso de aprendizaje, y: evaluación.

Análisis y diseño: comprenden el estudio de factibilidad; configuración curricular desde la docencia, la investigación y la proyección social; consideraciones organizacionales y de índole administrativo.

Concepción y desarrollo: corresponden a la construcción e implementación del proyecto académico y su inserción en el sistema educativo de la universidad.

Proceso de aprendizaje: está relacionado con el modelo pedagógico, medios tecnológicos y las metodologías utilizadas por el e-learning para llevarlo a cabo en los procesos sustantivos: docencia, investigación y proyección social.

Evaluación: corresponde a los procesos de mejoramiento, regulación del Ministerio de Educación Nacional, evaluación por pares y autoevaluación del proyecto.

\section{Aplicación de un modelo genérico de estándares}

En el contexto de Colombia la evaluación de calidad de un proyecto académico debe plantearse desde cinco dimensiones que encierran los dominios, tipos y entidades que expresa el modelo genérico de estándares: organización, procesos de enseñanza aprendizaje, desarrollo del e-learning, talento humano, e Infraestructura. Cada dimensión está subdividida en categorías o características que expresan referentes universales y particulares de la calidad que pueden tener aplicabilidad en cualquier institución o programa. Estas deben ser observables y valorables, para así poder emitir juicios o conceptos sobre su nivel de calidad.

Organización: esta dimensión se refiere a los procesos institucionales que dan soporte a los procesos de elearning. Se asume que en los procesos misionales, las políticas y los lineamientos estratégicos debe estar planeada y definida la estrategia de e-learning de la universidad que más adelante se desdoblará en los proyectos académicos que en esta modalidad emprenda la Institución. Comprende las siguientes categorías: misión, visión, lineamientos estratégicos, recursos físicos y financieros, gerencias, comunicación, políticas académicas y administrativas 
Procesos de enseñanza aprendizaje: la dimensión comprende los procesos de enseñanza aprendizaje en los que se plasma el proyecto educativo de un programa y contemplan las categorías: lineamientos pedagógicos, estructura curricular, investigación, proyección social y recursos virtuales.

Desarrollo del e-learning: hace referencia a la manera en que se vive el día a día del e-learning en la Universidad. Hace referencia a las categorías de: procesos de comunicación entre docentes y estudiantes, atributos del sitio web, fuentes de información y redes de conocimiento. nivel de cultura TIC, bienestar universitario, seguimiento y evaluación de los actores,

Talento Humano: en esta dimensión se tienen en cuenta las categorías de formación, capacitación y actualización de la planta docente y del grupo de apoyo que da soporte a todos los procesos de e-learning en la IES.

Infraestructura: tiene en cuenta todos los medios físicos y tecnológicos para llevar a cabo un proyecto de elearning. Abarca las categorías de: recursos físicos e informáticos, seguridad en todo nivel, mantenimiento y la plataforma de la gestión académica y financiera.

\section{Autoevaluación y Autorregulación}

Dentro de proceso de adaptación y adopción del modelo de referencia, resulta de vital importancia para su reconocimiento por el Ministerio Nacional de Colombia, que el proceso de evaluación del modelo sea identificado también con los procesos de autoevaluación y regulación de la educación superior, orientados por el Consejo Nacional de Acreditación (CNA, 2014). A la luz del decreto 1295 de 2010 (MEN, 2010), por el cual se reglamenta el registro calificado de que trata la Ley 1188 de 2008 y los decretos antes mencionados, han permitido que el CNA identifique factores centrales en el servicio educativo de educación superior (incluido el e-learning), los cuales son: misión y proyecto institucional, estudiantes, profesores, procesos académicos, bienestar institucional, organización, administración y gestión, egresados e impacto sobre el medio, y recursos físicos y financieros.

Cada factor tiene un conjunto de características que manifiestan los referentes universales y los particulares de la calidad y pueden ser aplicables a todo tipo de Institución o de programa académico; sin embargo, su lectura podrá ser diferenciada para dar cuenta de la diversidad de programas y de las especificidades que surgen de la existencia de diferentes tipos de Institución y de la individualidad de misiones y proyectos institucionales. Los factores mencionados tienen asociados indicadores que construye la Institución, con base en la información que el CNA (2006) debe evaluar. Con ellos se quiere establecer el cumplimiento de cada una de las características que han de apuntar, unos a aspectos centrales de la misma, otros a aspectos complementarios.

\section{Configuración del modelo de calidad}

El modelo de calidad para las IES, está compuesto por cinco elementos: una estructura de interoperabilidad, un modelo genérico de estándares, la adopción y adaptación de un modelo de referencia, un proceso de autoevaluación y regulación y una marca de calidad

Una estructura de interoperabilidad, que permita eficiencia y eficacia con la reutilización de materiales, metodologías y ambientes de aprendizaje (Dlouha y Burandt, 2015; Ehlers \& Hilera 2012). En términos de resultados las economías de escala brindan un indicador costo/beneficio más favorable.

Un modelo genérico de estándares, que asegure la interoperabilidad y el desarrollo de la calidad; que permita a través de modelos didácticos cumplir las expectativas del sujeto que aprende (estudiante) y que brinde los medios tecnológicos para el desarrollo de ambientes de aprendizaje adecuados (Islam, 2015; Ehlers \& Pawlowski, 2007).

La adopción y adaptación de un modelo de referencia, como ISO/IEC 19796, aceptado mundialmente y que contiene una estructura totalmente flexible y adaptable al e-learning.

Un proceso de autoevaluación y regulación que permita integrar estándares internacionales de calidad del e-learning, con la política, normatividad y legislación para las Instituciones de Educación Superior en Colombia.

Una marca de calidad que establezca ciertos niveles de certificación para procesos y productos, teniendo en cuenta para cada Institución sus características institucionales y contextuales.

El paso siguiente consistió en consolidar en cinco ejes propuestos, el modelo ya adaptado, partiendo de los componentes presentados en el modelo de adaptación y adopción: i) El desarrollo de la visión, políticas, estrategias, concienciación, identificación de actores y la definición de objetivos de calidad, corresponden al eje Modelo Organizacional; ii) La selección de métodos, instrumentos, métricas e indicadores corresponde al proceso de Normalización; iii) La participación, el uso y la evaluación que corresponden al eje Evaluación. 
El desarrollo continuo y el mejoramiento continuo se agrupan en el Desarrollo de la Calidad; y iv) La marca de calidad se equipara con el renacimiento nacional e internacional del programa por entes designados por la comunidad para ello.

\section{Modelo de calidad propuesto}

En la figura 2 se muestra el modelo resultante del proceso de configuración citado anteriormente. El modelo se explica a través de:

Modelo Organizacional: este componente le permite a la Institución trazar la ruta para que la visión del desarrollo del e-learning se explicite en la vida académica haciendo parte de la misión y de esta manera presentar su modalidad educativa, como una oportunidad que potencie el desarrollo académico de la Institución. Esto se logrará en la medida en que esta visión se pueda acoplar a todos los planes institucionales y sea factor integrador en la visión de la Institución y sus objetivos.

Desde el punto de vista estratégico el modelo privilegia aquellas iniciativas en las que los lineamientos del e-learning se relacionen con otras estrategias y políticas institucionales, para que puedan constituirse en un eje para el cambio en la organización. Esto conduce a que la gerencia del e-learning se inserte dentro del marco estratégico institucional y permita el direccionamiento claro, con una apropiada delegación de toma de decisiones operacionales. Desde el punto de vista más operacional se plantea la importancia de tener normatividad exclusiva para la modalidad, procesos administrativos y académicos documentados con soporte de TIC y a través de Internet, además de un sistema de administración del e-learning totalmente en línea. Desde la perspectiva financiera se sugiere la exploración y explotación de métodos innovadores de financiación para complementar la asignación institucional de presupuesto de para el e-learning y un plan financiero sostenible para todo el sistema.

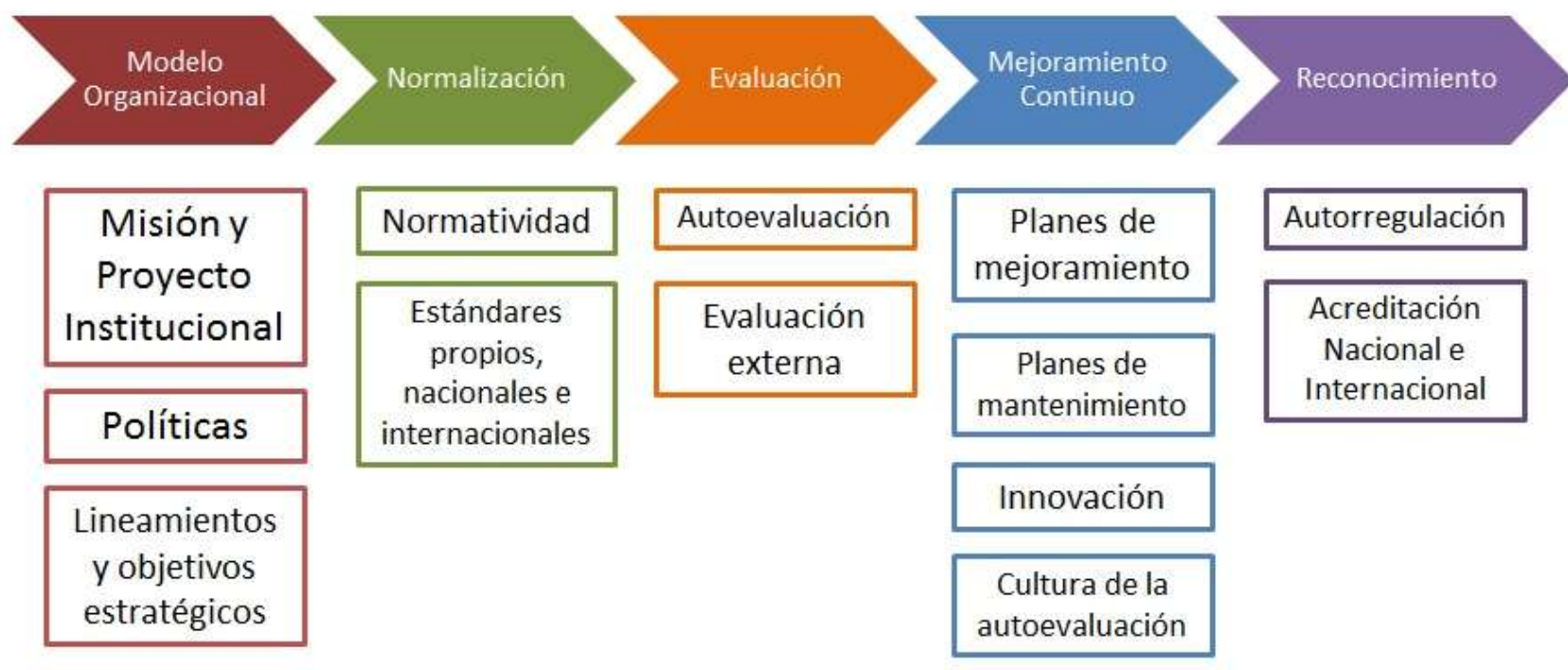

\begin{tabular}{|c|}
\hline Plan de \\
desarrollo \\
Institucional \\
\hline
\end{tabular}

Fig. 2: Modelo de calidad propuesto para las Instituciones de Educación Superior en Colombia

Normalización y Armonización: La normalización y armonización son un componente flexible dentro del modelo, que dependen de los objetivos de calidad de la Institución, los estándares propios y los que quiera acoger en el nivel internacional. En el caso de la normatividad se toma como un principio base y una condición sin la cual no puede haber un reconocimiento social de la calidad del programa.

Para el caso del proyecto se ha tomado como norma los decretos expedidos por el Ministerio de educación Nacional y el CNA para la acreditación de programas y acreditación institucional en su manera más global y general, adicionando aquellos elementos que particularizan enfoques disciplinares y profesionistas en un programa de e-learning. Respecto de los enfoques de estándares internacionales cada IES en Colombia es autónoma para tener en cuenta los elementos del modelo de apropiación y adaptación de la calidad del CEN, ISSS, QSEL y otros del estándar ISO 19796, que considere pertinentes y que esté armonizada con la normatividad mencionada anteriormente. De todos ellos puede construirse un conjunto de indicadores lo 
suficientemente amplio que desde la perspectiva del enfoque global de calidad abarca todos los procesos de un sistema de educación superior, los particulares de un programa o proyecto, los específicos de su modalidad (distancia y virtual) y los propios según su metodología.

La normalización y armonización permitió determinar 33 indicadores, agrupados en las cinco dimensiones del modelo genérico de estándares, como se presentó en la sección anterior, enmarcados dentro de la evaluación de la calidad de un proyecto de e-learning. Cada uno posee tres niveles (1, 2 y 3) que corresponden a un cumplimiento pobre, básico y superior.

Evaluación: El proceso de evaluación puede consolidarse en dos momentos. Uno es la autoevaluación, en la que la Institución asume el liderazgo y propicia la amplia participación y discusión de la comunidad universitaria, dado que la amplia participación permite una mirada interna de carácter holístico, menos subjetiva y matizada desde todas las perspectivas.

El otro momento es la evaluación externa concebida como la valoración de pares externos (individuos y entidades) de la calidad de una Institución o programa, de acuerdo con los lineamientos de calidad de la Institución, estándares de nivel internacional y la normatividad vigente. Esta revisión debe conducir a una declaración objetiva sobre la calidad del programa en concordancia con su organización, su funcionamiento y el cumplimiento de su función social. Esta va acompañada de recomendaciones para su mejoramiento, cuando haya discrepancias entre lo observado y lo que debe ser.

Los individuos $u$ organizaciones que realicen la evaluación externa deben emitir juicios rigurosos sobre la calidad, tomando como base el análisis de los conocimientos teóricos y metodológicos, habilidades que corresponden al saber y al saber-hacer propios de la comunidad que se desempeña en el campo de una determinada disciplina, profesión, ocupación u oficio y el modo como la Misión y el Proyecto Institucional se plasman en la formación de los estudiantes y se evidencian en los graduados. Por esto quienes practican una evaluación externa deben tener experticia en aspectos relevantes del saber específico del área correspondiente, o profesionales de áreas afines y debidamente capacitados en los procesos de autoevaluación, con conocimientos profundos en el uso y apropiación de las TIC.

Mejoramiento Continuo: La evaluación de la calidad no es un fin, sino un medio para determinar el grado de proximidad con el estado ideal o de cumplimiento máximo que determinan la armonización realizada previamente. Este proceso debe estar incluido dentro las políticas institucionales y reflejadas en cada uno de los proyectos y programas, integrado a los planes de desarrollo, promoviendo el cumplimiento de los enunciados misionales y de un ideal de excelencia. Debe intervenir permanentemente los procesos y alterar las dinámicas corporativas produciendo ajustes cuando las condiciones así lo indiquen. Esto solo es dable si existe arraigada una cultura de calidad y una clara comprensión de los procesos por parte de la comunidad académica (Ramírez, 2014; Ehlers, 2009).

Como estrategia de mejoramiento continuo deben diseñarse dos tipos de planes: i) de mantenimiento, parte de la comprensión de las fortalezas y están dirigidos a "mantenerlas", ii) de mejoramiento, que surge del reconocimiento de los aspectos donde la Institución tiene falencias y están orientados a "mejorarlas". Ambos planes se enmarcan dentro de las metodologías que la IES tiene para formularlos, se sugiere precisar para cada fortaleza o falencia: objetivo, acción, indicador, plazo, presupuesto y fecha de evaluación.

Otro elemento a tener en cuenta en el marco del mejoramiento continuo es la innovación, que se entiende como el proceso en el cual la Institución reconoce, adapta y adopta nuevos procesos como consecuencia de la conversión de una falencia en una fortaleza y la documenta para la gestión del conocimiento de la organización. En este sentido un elemento como el "Benchmarking" puede ser usado como estrategia de formación de fortalezas, debilidades y áreas de mejora a través de un proceso de colaboración (Ossiannilsson \& Landgren, 2012). La cultura de la calidad se evidencia cuando todos los elementos anteriores se incorporan como hábitos cotidianos dentro de la organización y hacen parte de la conciencia colectiva corporativa.

Reconocimiento: La etapa de reconocimiento se obtiene cuando organizaciones gubernamentales o no gubernamentales certifican la calidad del programa o la Institución. En el caso de Colombia, el Ministerio de Educación Nacional mediante una resolución certifica (acredita) la calidad con una vigencia que puede variar entre cuatro (4) y diez (10) años, dependiendo de la evaluación y el compromiso con el aseguramiento de la calidad. Para el caso de organizaciones no gubernamentales no existen procesos integrales para certificación de calidad avalados por el estado. Las IES se someten a la evaluación de estos entes sin prejuicio del reconocimiento o no del gobierno, para el caso de que lo hagan internacionalmente, ocurre de igual forma. 
El aspecto más importante de los contemplados en esta fase es la autorregulación, entendida como el proceso orientado al mejoramiento continuo a través de mecanismos que han sido apropiados por la comunidad académica y que son parte de la cotidianidad de la institución. En este orden de ideas una IES que se autorregula está en capacidad de cumplir a cabalidad su misión, impactando el entorno social en el que tiene influencia, dentro de un marco de mejoramiento continuo.

\section{El ciclo de calidad}

El mejoramiento continuo tiene como marco de referencia el ciclo de calidad. Los ciclos de calidad fueron implantados por primera vez en los años 60 , pero estos han ido evolucionando en la medida en que cada organización ha consolidado sus políticas de calidad.

En la figura 3 se plantea un ciclo de calidad para una IES en el entorno del modelo de calidad planteado en el trabajo. Los pilares del ciclo son la misión y el proyecto institucional, quienes marcan rumbo y fijan el objetivo estratégico, del cual se desprenden los objetivos estratégicos de calidad que se materializan en los planes de desarrollo que dan origen a los programas académicos, entre ellos los de e-learning. Aquí se inicia propiamente el ciclo de calidad.

El primer paso en este ciclo lo constituye la autoevaluación que responderá al modelo organizacional y a los procesos de armonización de estándares propia y a la normatividad vigente. Luego del proceso de autoevaluación se practica la evaluación externa con pares idóneos de acuerdo con el programa a evaluar. De estas dos evaluaciones y atendiendo las fortalezas y oportunidades de mejoramiento detectadas se trazan los planes de mantenimiento y mejoramiento que se evalúan permanentemente, lo que retroalimenta los planes de desarrollo y se inicia nuevamente el ciclo. Cabe anotar que la innovación es un componente diferenciador en la medida en que la Institución y los programas la vuelvan una práctica permanente de apropiación y cultura de la calidad.

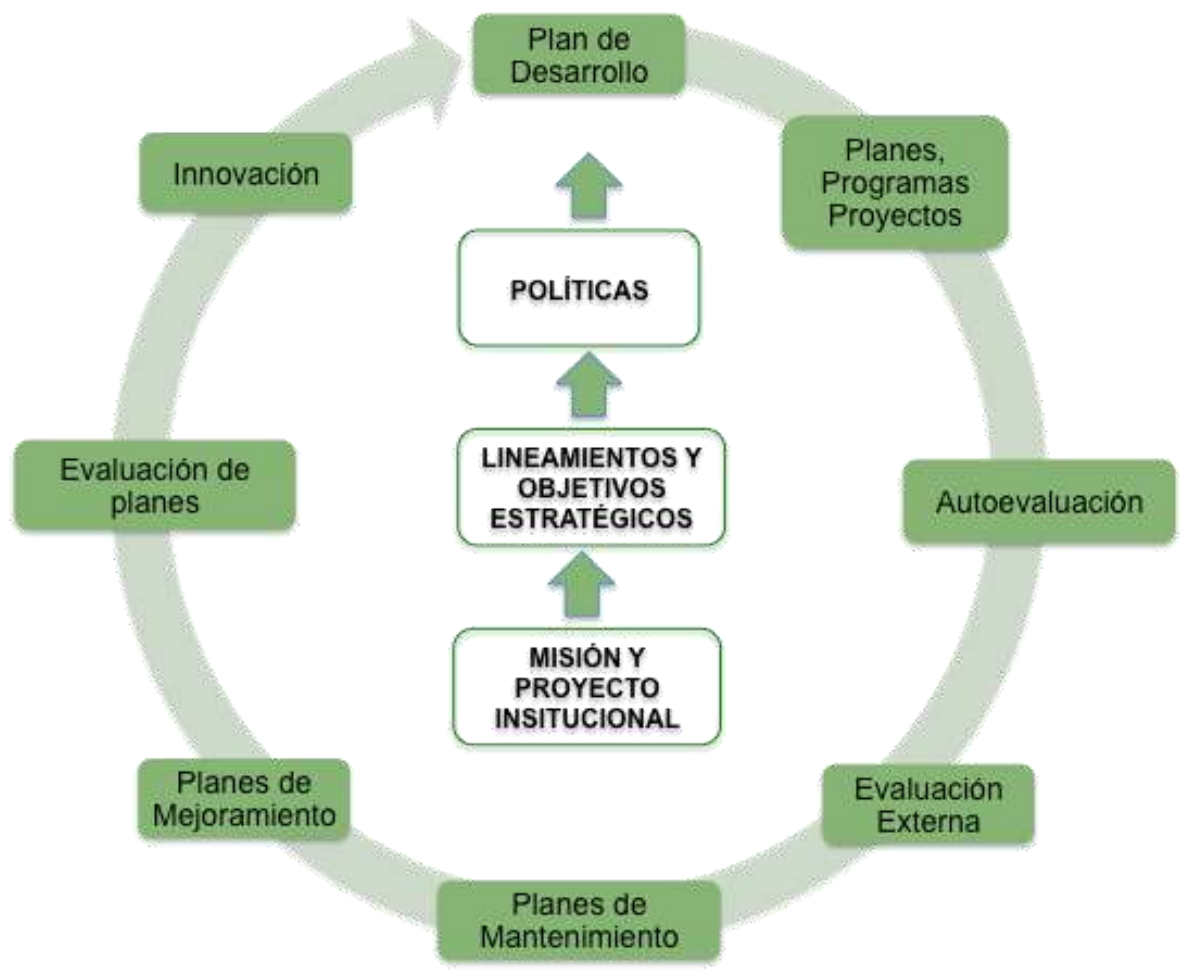

Fig. 3: Ciclo de calidad para la aplicación del modelo propuesto

El primer paso en este ciclo lo constituye la autoevaluación que responderá al modelo organizacional y a los procesos de armonización de estándares propia y a la normatividad vigente. Luego del proceso de autoevaluación se practica la evaluación externa con pares idóneos de acuerdo con el programa a evaluar. De estas dos evaluaciones y atendiendo las fortalezas y oportunidades de mejoramiento detectadas se trazan los planes de mantenimiento y mejoramiento que se evalúan permanentemente, lo que retroalimenta los planes de desarrollo y se inicia nuevamente el ciclo. Cabe anotar que la innovación es un componente diferenciador en la medida en que la Institución y los programas la vuelvan una práctica permanente de apropiación y cultura de la calidad. 
El modelo propuesto en el trabajo resulta útil por su aplicabilidad en el contexto del país, debido a que la gran mayoría de universidades colombianas se encuentra en el mismo nivel en cuanto a sus procesos de virtualidad; en muy pocos casos se tiene una visión de la calidad a largo plazo y como un todo en cuanto a la articulación entre lo académico y lo administrativo. La novedad del proyecto radicar en la articulación entre lo académico y lo administrativo, concebido como un todo frente a la estandarización y la homogenización en el concepto de calidad. Se pretende plantear el componente administrativo como un factor importante no solo en la toma de decisiones sino en la calidad del e-learning que se imparte.

\section{VALIDACIÓN DEL MODELO PROPUESTO}

Si bien es cierto que los estudios sobre la calidad del e-learning en Colombia no abundan, se han realizado algunos teniendo en cuenta parámetros de cobertura y penetración de las TIC, como el realizado en el año 2007 por el Ministerio de Educación Nacional, en el que se midió el impacto de las TIC y las concepciones de e-learning en el número de programas en esta modalidad. El estudio pretendió validar el modelo de calidad del e-learning planteado en IES de Colombia y hacer algunas recomendaciones a las instituciones sobre las dimensiones e indicadores considerados por dicho modelo, como elemento que aporte a la calidad.

\section{Metodología}

Se implementaron tres tipos de instrumentos: entrevistas, observación y un diagnóstico del estado del elearning que permitieron interactuar, conocer y comparar la calidad del mismo en algunas IES de Colombia. En la realización, ejecución y análisis de éstos instrumentos se tuvo en cuenta cinco categorías o aspectos indispensables que ayudaron a conocer y comparar la situación actual de algunas universidades reconocidas del país y obtener una visión global de cómo se está implementando el e-learning en las IES. Las dimensiones que se tuvieron en cuenta fueron las mismas del modelo propuesto: aspectos organizacionales, procesos de enseñanza y aprendizaje, desarrollo del e-learning, talento humano e infraestructura tecnológica.

El diagnóstico se aplicó a través de una plataforma virtual, donde directivos o docentes encargados del campus virtual de cada universidad hicieron el registro de sus datos para tener acceso a ella; como valor agregado el sistema propone recomendaciones para el mejoramiento de la calidad del e-learning en la Institución; además de una gráfica que daba a conocer una visión general de lo que respondía cada Universidad.

Para cada uno de los indicadores se desarrollaron preguntas que se calificaron en la siguiente escala de valores: (5) se cumple plenamente, (4) se cumple en alto grado, (3) se cumple aceptablemente, (2) se cumple insatisfactoriamente y (1) no se cumple, N/A no aplica.

El diagnóstico tiene dos fases: i) Aplicación del cuestionario: se debe ingresar en la web creando una cuenta de usuario y asociarlo a la IES que representa; ii) Diagnóstico propiamente dicho: una vez ingresados los datos de acuerdo con los parámetros del modelo de calidad, el sistema arroja un diagrama circular, en el que se muestran las fortalezas y las oportunidades de mejoramiento de la institución. Al mismo tiempo el sistema le entrega a la IES un reporte con sugerencias para un plan de mejoramiento, que se origina en el cumplimiento o no, de los niveles de los indicadores de calidad del modelo.

La Entrevista además de un acercamiento personal con los responsables del e-learning, sirvió como apoyo a dudas o inquietudes en el diagnóstico. La observación directa en la Institución sirvió como elemento adicional que contrasta la información y el diagnóstico.

\section{Ficha de la muestra:}

Objetivo del estudio: validar el modelo de calidad del e-learning propuesto en el proyecto, mediante la aplicación de una encuesta, una entrevista y observación directa en campo.

Población Objetivo: Instituciones de Educación Superior de Colombia con oferta de programas en modalidad e-learning.

Marco Muestral: Instituciones de Educación superior de Colombia, que están en la base de datos del Sistema Nacional de Instituciones de Educación Superior SNIES(2015).

Método de Muestreo: En el diseño se empleó una muestra aleatoria dentro de las diez (10) principales ciudades del país.

Tamaño de la Muestra: quince por ciento (15\%) del marco muestral.

Representatividad de la muestra: se tomaron instituciones de educación superior de las diez (10) principales ciudades del país, con programas de e-learning que agrupan la mayoría de la oferta educativa en esta modalidad.

Error: es del cinco por ciento (5\%), dentro de un intervalo de confianza del 95\% 


\section{Recolección y análisis de la información}

El total de IES clasificadas como universidades en el Sistema Nacional de Información de la Educación Superior (SNIES) es de 133 instituciones. Se escogieron para la validación del modelo 2 universidades aleatoriamente de cada una de las 10 principales ciudades de Colombia que tuvieran programas o cursos en modalidad virtual. El análisis del diagnóstico practicado a las IES y un resumen de las valoraciones obtenidas en cada uno de los indicadores se muestran en la tabla 1.

\section{Discusión de los resultados}

Para poner en perspectiva los análisis realizados, es importante tener en cuenta que según el SNIES, existen 11743 programas activos, de los cuales 460 tienen modalidad virtual, equivalente al $4 \%$ de la oferta académica. En las Universidades existen 231 programas en esta modalidad lo que equivale al $2 \%$ de tal oferta, y de estos $123(53 \%)$ son de pregrado y el resto $108(47 \%)$ de posgrado, como se observa, en el momento existen más programas activos de pregrado que de posgrado en modalidad virtual.

En términos generales puede hablarse de una brecha entre universidades que tienen sistemas de e-learning altamente desarrollados en todas sus dimensiones y otras que tienen sistemas de educación virtual menos evolucionados y que deben hacer grandes esfuerzos en políticas, planeación e inversiones en el área para reducir una diferencia que puede crecer de manera acelerada si no se toman acciones en el corto, mediano y largo plazo. Desde la dimensión organizacional la validación permitió concluir que los procesos del elearning en las instituciones de educación superior colombianas se encuentran desarticulados de los procesos misionales, debido a que en su gran mayoría, estos nacen de iniciativas particulares y nunca como producto de una reflexión de la filosofía institucional y la revisión de los lineamientos estratégicos, planes de desarrollo y programas en el largo plazo.

Es importante tener en cuenta que en la gran mayoría de las instituciones no se ha consolidado un sistema de administración del e-learning totalmente en línea. Como evidencia de lo expuesto, se encontró que solo la mitad de las universidades ha realizado un proceso de interiorización y sensibilización sobre lo que significa un proyecto de e-learning. En este mismo sentido el nivel de empoderamiento en las instancias directivas sobre este tipo de proyectos y los procesos de comunicación de la visión en muy pocos casos han estado estructurados y debidamente realizados.

Casi todas las Universidades carecen de indicadores que den cuenta del mejoramiento permanente de los lineamientos académicos para el e-learning, esta es una conclusión importante para la implementación de un modelo de calidad como el propuesto. En las IES consultadas, el uso de las redes sociales es deficiente, por esto no se han introducido como medios de aprendizaje y comunicación, pues hoy son las que marcan tendencia en los sistemas de interacción grupal.

Respecto de la producción de recursos y ambientes educativos las fases más relevantes en el diseño y la producción tienen que ver con la revisión de fuentes para derechos de autor, comparación entre los objetivos de aprendizaje y los del curso a realizar, selección de metodologías y estrategias tecnológicas, primando los estilos de aprendizaje. Es de resaltar que no se encontraron procesos consistentes con una norma o estándar internacional, sin embargo, la mitad de las IES que participaron en el diagnóstico manifiestan tener estándares diseñados y debidamente documentados. Tampoco cuentan con un manual de buenas prácticas asociadas a las metodologías de diseño, producción y montaje de recursos y ambientes educativos.

Sobre las políticas de propiedad intelectual y derechos autor, aunque puede afirmarse que todas cuentan con un reglamento, no tienen procedimientos establecidos y personal necesario para la revisión y cumplimiento de tal normatividad. No se evidencia la aplicación de estándares internacionales para estos procedimientos. No es frecuente el uso de licencias creative commons. Es importante destacar que la mayoría de las IES realizan cursos de formación y actualización sobre el uso de herramientas tecnológicas y plataformas de gestión de e-learning LMS (Learning Management System), para estudiantes, administrativos y docentes. Se ofrecen algunos diplomados o capacitaciones, pero no se orientan con la frecuencia óptima para los propósitos de la estrategia de esta metodología. 
Tabla 1: Distribución porcentual de las respuestas para cada categoría, una vez aplicados los instrumentos para la recolección de la información.

\begin{tabular}{|c|c|c|c|c|c|c|}
\hline Dimensiones & Categorías & 1 & 2 & 3 & 4 & 5 \\
\hline \multirow{12}{*}{$\begin{array}{l}\text { 1. Aspectos } \\
\text { organizacionales }\end{array}$} & 1.1 Visión para el desarrollo del e-learning en la organización. & $0 \%$ & $0 \%$ & $17 \%$ & $28 \%$ & $56 \%$ \\
\hline & 1.2 Comunicación de la visión en la organización & $0 \%$ & $11 \%$ & $28 \%$ & $44 \%$ & $17 \%$ \\
\hline & 1.3 Estrategia de e-learning & $0 \%$ & $0 \%$ & $39 \%$ & $28 \%$ & $33 \%$ \\
\hline & $\begin{array}{l}\text { 1.4 Relación de la estrategia e-learning con los demás planes de } \\
\text { la organización }\end{array}$ & $0 \%$ & $0 \%$ & $42 \%$ & $33 \%$ & $25 \%$ \\
\hline & 1.5 Gerencia estratégica del e-learning & $0 \%$ & $6 \%$ & $33 \%$ & $44 \%$ & $17 \%$ \\
\hline & $\begin{array}{l}1.6 \text { Coherencia con los marcos estratégicos, planes y proyectos } \\
\text { locales, regionales y nacionales. }\end{array}$ & $0 \%$ & $0 \%$ & $25 \%$ & $42 \%$ & $33 \%$ \\
\hline & 1.7 Políticas académicas y administrativas para el e-learning & $0 \%$ & $17 \%$ & $17 \%$ & $33 \%$ & $33 \%$ \\
\hline & 1.8 Reglamentos para programas de e-learning. & $0 \%$ & $0 \%$ & $17 \%$ & $17 \%$ & $67 \%$ \\
\hline & 1.9 Procesos organizativos de apoyo & $0 \%$ & $0 \%$ & $17 \%$ & $33 \%$ & $50 \%$ \\
\hline & 1.10 Recursos económicos para el e-learning. & $0 \%$ & $0 \%$ & $0 \%$ & $75 \%$ & $25 \%$ \\
\hline & 2.1 Criterios o lineamientos pedagógicos para el e-learning. & & & & $100 \%$ & $0 \%$ \\
\hline & 2.2 Estructura curricular & $0 \%$ & $0 \%$ & $0 \%$ & $50 \%$ & $50 \%$ \\
\hline
\end{tabular}

2. Procesos de enseñanza y aprendizaje
2.3 Organización del equipo para el diseño y producción de recursos educativos para la web y ambientes virtuales de aprendizaje (AVAs)

2.4 Procesos de diseño y producción de recursos para el aprendizaje.

2.5 Disponibilidad de recursos electrónicos para el aprendizaje

2.6 Software específico para el diseño y producción de recursos educativos.

3.1 Comunicación electrónica entre los distintos actores (estudiantes, profesores, directivos, administrativos y otras comunidades).

\begin{tabular}{ccccc}
$0 \%$ & $0 \%$ & $17 \%$ & $0 \%$ & $83 \%$ \\
\hline $0 \%$ & $0 \%$ & $17 \%$ & $50 \%$ & $33 \%$ \\
\hline $0 \%$ & $0 \%$ & $0 \%$ & $50 \%$ & $50 \%$ \\
\hline & & & $17 \%$ & $83 \%$ \\
\hline
\end{tabular}

$\mathrm{N} / \mathrm{A}$

3. Desarrollo del Sensibilización hacia el e-learning y habilidades de manejo de
TICs entre los distintos actores.

$\begin{array}{llllll}3.2 \text { Disponibilidad y Funcionalidad del sitio web. } & 0 \% & 0 \% & 0 \% & 59 \% & 41 \%\end{array}$

e-learning

$\begin{array}{lllll}0 \% & 8 \% & 7 \% & 45 \% & 40 \%\end{array}$

3.4 Acceso a diversas fuentes de información y redes de conocimiento.

$6 \% \quad 0 \% \quad 0 \% \quad 50 \% \quad 44 \%$

3.5 Seguimiento a progreso y logros del estudiante y a la acción docente.

3.6 Seguimiento al logro de estudiantes y al logro docente

$\begin{array}{lllll}0 \% & 0 \% & 0 \% & 75 \% & 25 \%\end{array}$

4.1 Formación docente en diseño y docencia por medio de ambientes virtuales de aprendizaje.

$50 \% \quad 50 \%$

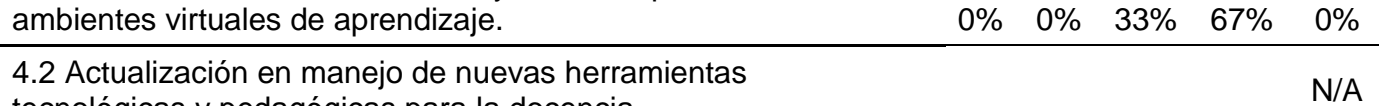

4. Talento humano tecnológicas y pedagógicas para la docencia

$\begin{array}{lllllll}4.3 \text { Planes de capacitación docente en e-learning } & 0 \% & 0 \% & 17 \% & 67 \% & 17 \%\end{array}$

$\begin{array}{lllllll}4.4 \text { Capacitación y seguimiento al personal de apoyo. } & 0 \% & 16 \% & 50 \% & 17 \% & 17 \%\end{array}$

5.1 Infraestructura de Telecomunicaciones $\quad$ N/A

5. 2 Equipo de cómputo disponible para los servicios web y N/A

sistemas de respaldo

\begin{tabular}{ll}
\hline 5.3 Ancho de banda disponible para conexión a Internet & N/A
\end{tabular}

5.4 Sistemas de seguridad a servicios web contra intrusos y fallos

5. Infraestructura de sistemas.

\begin{tabular}{llll}
\hline $\begin{array}{l}5.5 \text { Personal de Mantenimiento y administración de instalaciones } \\
\text { locativas, aplicaciones y centro de computo }\end{array}$ & $0 \% \quad 0 \% \quad 0 \% \quad 50 \%$ & $50 \%$ \\
\hline 5.6 Disponibilidad de servicios de red e Internet. & N/A \\
\hline 5.7 Disponibilidad de recursos de cómputo. & N/A \\
\hline 5.8 Sistema de Gestión del aprendizaje, Académico y Financiero & N/A \\
\hline
\end{tabular}


En cuanto al acceso a diferentes redes de información y conocimiento, un número muy reducido de instituciones pertenecen a redes virtuales de conocimiento en el área de programas virtualizados, donde los docentes y estudiantes accedan a ellas. El seguimiento y evaluación de los estudiantes se realiza a través la plataforma LMS con diferentes estrategias, pero en la mayoría de los casos este es un punto que está en discusión en cada Institución por la diversidad de conceptos y metodologías sobre estos procesos. No se evidenció la existencia de búsquedas semánticas, conexiones a redes académicas y servicios de búsqueda especializados en bases de datos internacionales.

Con respecto a la infraestructura tecnológica se puede afirmar que las instituciones cuentan con el acondicionamiento básico como centro de comunicaciones: redes eléctricas reguladas y normales, elementos contra incendios, centro de cableado eléctrico y estructurado, y sistema de tierras; todos con normas IEEE, TIA, además del personal de mantenimiento y administración, pero no cuenta con infraestructura suficiente para e-learning.

La siguiente infografía (figura 4) muestra los aspectos por mejorar en el e-learning de las universidades colombianas, en cada una de las dimensiones del modelo de e-learning.

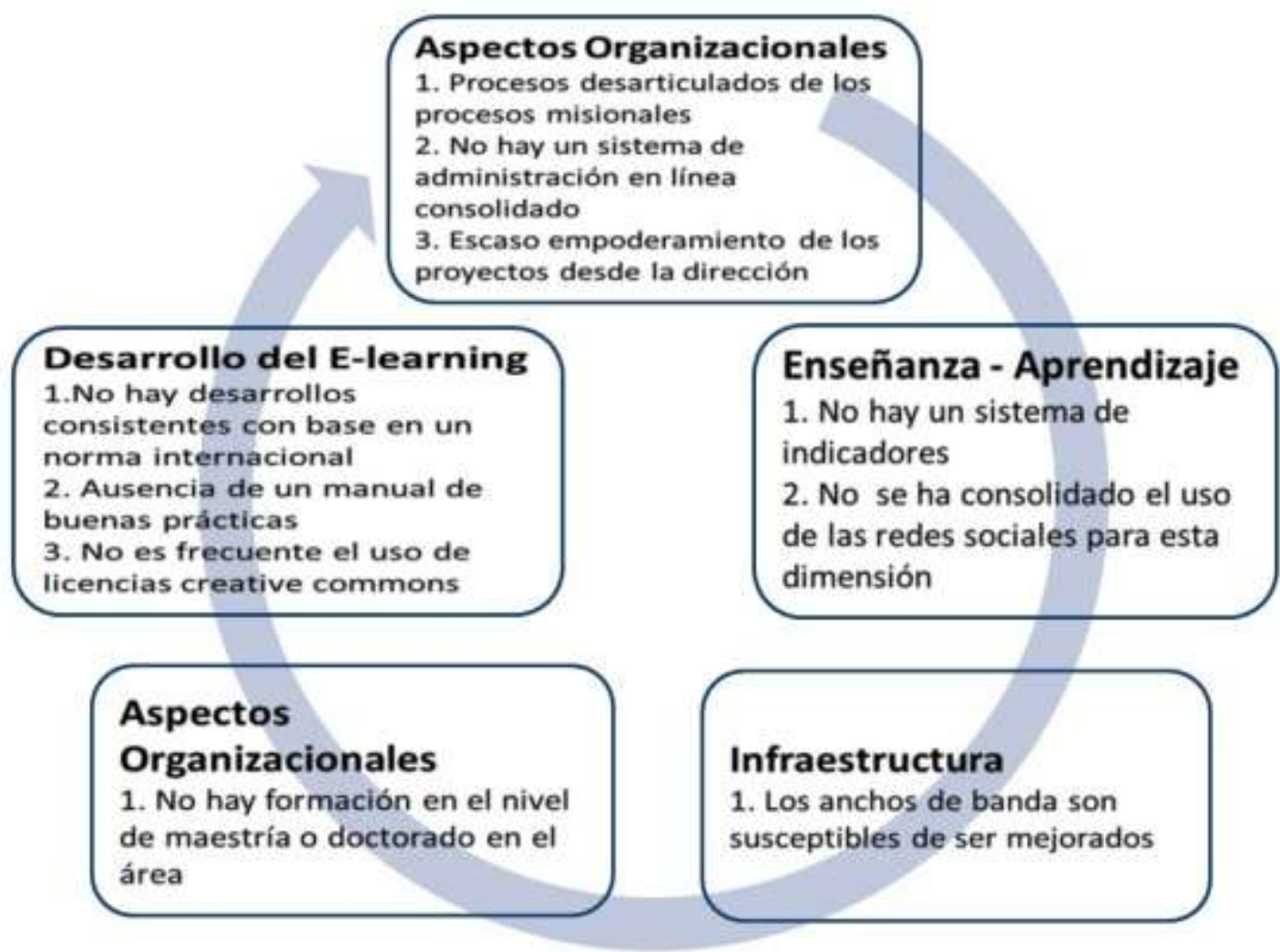

Fig. 4: Infografía. Aspectos por mejorar en el e-learning de las universidades

\section{CONCLUSIONES}

La aplicación del modelo permite detectar una brecha entre universidades que tienen sistemas de e-learning desarrollados en todas sus dimensiones y otras que tienen sistemas de educación virtual menos evolucionados y que deben hacer grandes esfuerzos en políticas, planeación e inversiones en el área para reducir una diferencia que puede crecer de manera acelerada si no se toman acciones en el corto, mediano y largo plazo.

Los procesos del e-learning en las instituciones de educación superior colombianas se encuentran desarticulados de los procesos misionales, debido a que en su gran mayoría, estos nacen de iniciativas particulares y nunca como producto de una reflexión de la filosofía institucional y la revisión de los lineamientos estratégicos, planes de desarrollo y programas en el largo plazo.

Un elemento que debe llamar a la reflexión en las IES es el reducido número que pertenecen o tienen acceso a diferentes redes virtuales de información y conocimiento en áreas de interés, en las que los docentes y estudiantes tengan participación. Los avances en infraestructura y tecnología se han 
desarrollado de manera importante y en algunos momentos priman sobre otros aspectos del proceso de formación virtual, cubriendo falencias en aspectos como los comunicacionales y la evaluación de estudiantes y profesores.

Aunque todas las instituciones tienen concebido un plan de aseguramiento de la calidad, ninguna incorpora un modelo como los estudiados en la investigación, más bien toman aspectos de muchos de ellos y los incorporan en dicho plan, sin ningún criterio establecido. Ninguna de las instituciones consultadas hizo o está en proceso de armonización o construcción de estándares para el e-learning y más bien están construyendo indicadores que dan cuenta de los objetivos estratégicos trazados al respecto. El modelo es consistente porque tiene en cuenta diferentes aspectos de los procesos de formación, no solo desde los procesos tanto de enseñanza como de aprendizaje, sino también los aspectos comunicativos, organizacionales y tecnológicos; que en muchos de los modelos consultados no se tocan, ya que tienen un interés particular en área específica del proceso.

\section{AGRADECIMIENTOS}

Este trabajo ha sido financiado parcialmente por el Programa de Investigación: Construcción de ambientes innovadores e inclusivos para el aprendizaje en escenarios virtuales, financiado por el Departamento Administrativo de Ciencia y Tecnología de Colombia (COLCIENCIAS) y forma parte de la Tesis Doctoral: "Modelo de Calidad del e-learning para IES colombianas", para optar por el título de Doctor en Ingeniería Informática de la Universidad Pontificia de Salamanca (España) de uno de los investigadores.

\section{REFERENCIAS}

Barberá, E. Calidad de la enseñanza 2.0. Retrieved may0 152010 from RED, Revista de Educación a Distancia: http://www.um.es/ead/red/Mo (2008)

Bates, T. Calidad y aprendizaje virtual: el desafío de las instituciones de e-learning. Quality: state of the art and trends. Bogotá: Ministerio de Educación Nacional de Colombia. (2007)

Cardona, D. y Sánchez, J., Indicadores Básicos para Evaluar el Proceso de Aprendizaje en Estudiantes de Educación a Distancia en Ambiente e-learning. ISSN 0718-5006 Form. Univ 3(6) 15-32 (2010)

Consejo Nacional de Acreditación.. CNA. From www.cna.gov.co (diciembre, 2014)

Consejo Nacional de Acreditación. Indicadores para la autoevaluación con fines de acreditación de programas de pregrado en las modalidades a distancia y virtual. CORCAS Editores. (2006)

De la Vega, L., Hacia un marco analítico y metodológico para la evaluación del mejoramiento educativo en escuelas chilenas. ISSN 0718-4565 Calidad en la educación, 42, 61-91 (2015)

Dlouha, J. y Burandt, S., Design and evaluation of learning processes in an international sustainability oriented study programme. In search of a new educational quality and assessment method. doi:10.1016/j.jclepro.2014.09.096, Journal of Cleaner Production. 106(1) 247-258 ( 2015)

Donabedian, A. TheDefinition of Quality and ApproachestoltsAssessment. 1. (1980)

Dondi, C. La calidad de la experiencia de aprendizaje como factor discriminante en el desarrollo del potencial de las TIC en los sistemas educativos y formativos. Retrieved April 22010 from Universidad Politécnica de Madrid: http://serviciosgate.upm.es/ojs/index.php/relada/article/viewFile/27/27_(Enero, 2008)

Duart, J. M., y Lupiáñez, F. La gestión y la administración del e-learning en la universidad. Revista de Universidad y Sociedad del Conocimiento, 2 (1), 100-105. (2005)

EFQUEL. Consultado en http://cdn.efquel.org/wp-content/uploads/2012/03/GP2.pdf (2008)

Ehlers, D., y Pawlowski, J. The Quality Adaptation Model: adoption and adaptation of the quality standard ISO/IEC 19796-1, learning, training and education. Educational Technology and Society, 2 (10), 3-16. (2007)

Ehlers, D., y Pawlowski, J. Handbook on quality and standardization in e-learning. Springer. (2006)

Ehlers, U. Understanding quality culture. Quality Assurance in Education (17), 343-363. (2009)

Ehlers, U., y Hilera, J. Special issue on quality in e-learning. Journal of ComputerAssisted Learning,, 28 (12), 1-3. (2012) 
Hilera, J. R., y Hoya, R., Estándares de E-learning: Guía de Consulta. Universidad de Alcalá. (2010)

Islam, A.K.M. Najmul. E-learning system use and its outcomes: Moderating role of perceived compatibility, doi: 10.1016/j.tele.2015.06.010 Telematics and Informatics. 33(1), 48-55 (2016)

Martínez, E. y otros 3 autores, An application of the performance-evaluation model for e-learning quality in higher education. doi: 10.1080/14783363.2013.867607. Total Quality Management \& Business Excellence. 26 (5-6) 632-647 (2015)

Ministerio de Educación Nacional de Colombia. Decreto Ley 1295 de 2010.Bogota: MEN. (2010)

Ministerio de Educación Nacional de Colombia. Estudio: Modelos virtuales en las IES Colombianas. MEN. Consultado en http://www.colombiaaprende.edu.co/html/mediateca/1607/articles-126604_archivo.pdf (2007)

Ossiannilsson, E., y Landgren, L. Quality in e-learning - a conceptual framework based on experiences from three international benchmarking projectsjcal_439. Quality enhancement on e-learning. (2012)

Pawlowski, J., Global Learning Quality. Quality of Higher Education, 5, 12-31. (2008)

Pawlowski, J., Quality mark e-learning: developing process- and product-oriented quality for learning, education and training. Int. J. Learning Technology, 3 (1), 51-71. (2007)

Pons, D.; Ramon H., J.; Fernández, L., Managing the quality of e-learning resources in repositories. doi: 10.1002/cae.21619 Computer Applications in Engineering Education. 23(4), 477-488 (2015)

Ramírez, D., Educación a distancia y políticas públicas en materia de telecentros digitales: el caso del proyecto "CASA" de México. ISSN 0719-367X Cuad.inf. 35, 55-67 (2014)

Sandoval Yáñez, C., Propuesta para implementar un sistema de gestión del conocimiento que apoye el diseño de un curso online. ISSN 0718-3305 Ingeniare. Rev. chil. ing., 21 (3) 457-471 (2013)

Sangrá, A. La calidad de las experiencias virtuales de educación superior. Red Digital. Revista de Tecnologías de la Información y la Comunicación Educativas (6). (2001)

SNIES. Sistema Nacional de Información de la Educación Superior. Consultado: http://www.mineducacion.gov.co/sistemasdeinformacion/1735/w3-propertyname-2672.html (2015)

Stracke, C. Categories and Purposes of (e-Learning) Standards: The Need for Harmonization of Interoperability and Quality Development. Future of Interoperability Standard Meeting 2010. Manchester. (2010)

Stracke, C. The Quality Initiative E-Learning in Germany. Desarrollo de la calidad y Estándares de calidad para e-Learning. Seminario de Calidad del E-learning. Bogotá: Ministerio de Educación Nacional de Colombia. (2007)

Stracke, C. Interoperability and Quality Development in e-Learning: Overview and Reference Model for eLearning Standards. Proceedings of the Asia-Europe e-Learning Colloquy. Seoul. (2006) 